\title{
Autonomous Tracking of a Ground Vehicle by a UAV
}

\author{
Kartik B. Ariyur and Kingsley O. Fregene
}

\begin{abstract}
We propose a generally applicable method for the tracking of ground vehicles by aerial vehicles. The method does not require any modifications to the guidance and control of the UAV. It only requires the capability to follow waypoint commands. Sensing of ground vehicle position with significant time delays is assumed. The delays model the time of image processing, and the communication delays involved in sending data to a ground station, performing the computations and receiving the results on the UAV. Variants of the method have been tested successfully on the field and may see widespread deployment.
\end{abstract}

\section{INTRODUCTION}

Tracking a moving (ground) target from a UAV is a key requirement for many reconnaissance, surveillance and target acquisition (RSTA) application - one of the many uses of UAVs. For RSTA purposes, the UAVs are equipped with appropriate sensor payloads to enable them perform their desired mission. In many cases, the track sensors consist of cameras that are mounted so that they point forward or sideways. The tracking problem is typically to autonomously and dynamically position the UAV so that a moving target remains substantially within the vehicle's camera field of view regardless of the specific target motion patterns. The problem is a special case of a predator-prey differential game, which has a vast literature starting from the pioneering work of Isaacs [1], and cannot be fully referenced herein. We mention only a couple of recent works. In [2], a variety of path planning methods was described for UAVs to track targets moving at varying speeds. A tracking scheme was described in [4] for UAVs that operate in communication-constrained adversarial environments.

In this work, we describe a system for tracking a ground target from UAVs with rotary wing and fixed wing airframes via delayed estimation of target motion from noisy sensors. The algorithm works by estimating the acceleration of the ground vehicle and using a point mass model to estimate its velocity and position; these estimates are used to produce waypoints for the UAV.

The beauty of this approach is that the only information provided to the UAV guidance system are waypoints required to establish and maintain track of the target. Therefore, no modification of any sort is required on the UAV's guidance and control systems. As long as the vehicle can follow simple waypoint commands in closed loop, it will work with the proposed method. Another advantage is that it works very well for UAVs that have sensors (e.g. cameras) of fixed attitude and geometry. The problem would be somewhat simpler if the camera is steerable or pointable (and that is easily accommodated in our scheme). In addition to tracking from rotary wing UAVs, a key feature of the approach described in this work is that it allows tracking with fixed camera attitude/geometry from small fixed wing UAVs that are minimally maneuverable. Many of the UAVs have replaceable sensors, so the camera may be replaced by an infra red camera or a millimeter wave radar depending on the environmental conditions of the mission.

This work was supported by the DARPA SEC program

Kartik B. Ariyur, (kartik.ariyur@honeywell.com) is with the Navigation group, and Kingsley O. Fregene, (kingsley.fregene@honeywell.com) is with the High Integrity Flight Controls group in Honeywell Aerospace Advanced Technology, 3660 Technology Drive, Minneapolis, MN, 55418.

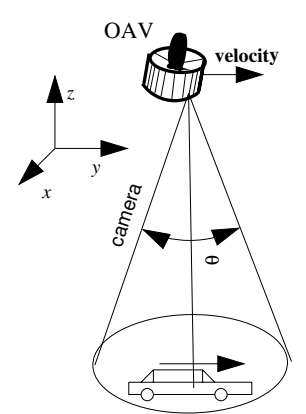

Fig. 1. Aerial tracking of a ground vehicle

The rest of this work is organized as follows. Section II formulated the problem, section III presents the basic modeling, section IV the generation of waypoints for stable tracking, and section $\mathrm{V}$ presents the results.

\section{Problem OvervieW}

The delay and noise inherent in visual recognition and tracking algorithms, especially when added to communication delays makes our tracking problem difficult. Moreover, if there are minimum speed limits on the aerial vehicle (as any fixed wing UAV would have), the ground vehicle can easily give the slip to the tracking UAV. We pose the problem as the tracking of a two dimensional double integrator point mass model with a three dimensional double integrator point mass model with velocity and acceleration constraints. We model the camera sensor as being able to maintain target detection within a right circular cone vertically beneath the UAV with the cone angle being equal to the field of view (FOV) $\alpha$ of the camera (this is illustrated in Figure 1). The FOV circle on the ground thus has a radius of $z \tan \frac{\alpha}{2}$ where $z$ is the altitude of the vehicle. We propose tracking control laws that are exponentially stable and maintain stable tracking even with extremely noisy tracking sensors. This structure abstracts the essential features of the tracking problem without the distractions of detailed vehicle dynamics and various constraints. Furthermore, it eases tracking design for UAVs whose attitude stabilization control laws (commonly known as the inner loop) are already implemented, and therefore a given.

\section{CHASER AND PREY MODELS}

We work purely with discretized models as the handling of delays is natural in this setting. We denote the sampling time with $T, \mathbf{x}_{p}$, and $v_{p}$ denote planar position $\left(x_{p}, y_{p}\right)$ and velocity vectors of the quarry, and $\mathbf{x}_{c}, \mathbf{v}_{c}$ denote the three dimensional position $\left(x_{c}, y_{c}, z_{c}\right)$ and velocity vectors of the chaser. The prey model is simply a double integrator with an unknown acceleration input $\mathbf{a}_{p}$ :

$$
\begin{aligned}
& \mathbf{x}_{p}(k+1)=\mathbf{x}_{p}(k)+T \mathbf{v}_{p}(k) \\
& \mathbf{v}_{p}(k+1)=\mathbf{v}_{p}(k)+T \mathbf{a}_{p}(k)
\end{aligned}
$$

The chaser model incorporates information about the position tracking and velocity tracking time constants $\left(\tau_{x}\right.$ and $\left.\tau_{v}\right)$ of the 
inner loop controller on board the UAV:

$$
\begin{aligned}
\mathbf{x}_{c}(k+1)= & \mathbf{x}_{c}(k)+T \mathbf{v}_{c}(k) \\
\mathbf{v}_{c}(k+1)= & -\frac{T}{\tau_{x} \tau_{v}} \mathbf{x}_{c}(k)+\left(1-\frac{T}{\tau_{v}}\right) \mathbf{v}_{c}(k) \\
& +\frac{T}{\tau_{x} \tau_{v}} \mathbf{x}_{c}^{r e f}(k),
\end{aligned}
$$

where $\mathbf{x}_{c}^{r e f}(k)$ is the tracking set point. We next write the equation for the planar position error between the chaser and the prey. The planar component of the chaser position and velocity are denoted respectively by $\mathbf{x}_{c}^{p l}$ and $\mathbf{v}_{c}^{p l}$ :

$$
\begin{aligned}
\delta \mathbf{x}_{p l} \equiv & \mathbf{x}_{c}^{p l}-\mathbf{x}_{p} \\
\delta \mathbf{v}_{p l} \equiv & \mathbf{v}_{c}^{p l}-\mathbf{v}_{p} \\
\delta \mathbf{x}_{p l}(k+1)= & \delta \mathbf{x}_{p l}(k)+T \delta \mathbf{v}_{p l}(k) \\
\delta \mathbf{v}_{p l}(k+1)= & -\frac{T}{\tau_{x} \tau_{v}} \delta \mathbf{x}_{p l}(k)+\left(1-\frac{T}{\tau_{v}}\right) \delta \mathbf{v}_{p l}(k) \\
& -\frac{T}{\tau_{x} \tau_{v}} \mathbf{x}_{p}(k)-\frac{T}{\tau_{v}} \mathbf{v}_{p}(k)-T \mathbf{a}_{p}(k) \\
& +\frac{T}{\tau_{x} \tau_{v}} \mathbf{x}_{c}^{r e f, p l}(k),
\end{aligned}
$$

where $\mathbf{x}_{c}^{r e f, p l}(k)$ is the planar part of the chaser position set point.

\section{The Tracking Control LaW}

If we can set the tracking set point to cancel the terms arising from prey vehicle position, velocity, and acceleration in the error equation above, we will have exponential tracking of the quarry. The control law in this case would be-

$$
\mathbf{x}_{c}^{r e f, p l}(k)=\frac{T}{\tau_{x} \tau_{v}} \mathbf{x}_{p}(k)+\frac{T}{\tau_{v}} \mathbf{v}_{p}(k)+\tau_{x} \tau_{v} \mathbf{a}_{p}(k)
$$

However, we have to work from delayed and noisy measurements of the quarry position and velocity. To this end, we estimate current quarry position, velocity and acceleration from the measurements. We assume that the delay $(n T)$ is an integral multiple of the sampling time $T$-this is realistic since the sampling time is small compared to the delay. The measurements we have are

$$
\begin{aligned}
& \mathbf{x}_{p}^{\text {meas }}(k)=\mathbf{x}_{p}(k-n)+v_{1} \\
& \mathbf{v}_{p}^{\text {meas }}(k)=\mathbf{v}_{p}(k-n)+v_{2},
\end{aligned}
$$

where $v_{1}$ and $v_{2}$ represent measurement noise, whose properties under different operating conditions may be available. The estimation of quarry dynamics needs some filter/predictor. To avoid making assumptions upon the measurement noise in estimator design, we design an FIR (finite impulse response) filter to this end. The filter simply takes a weighted average of the $m$ past estimates of acceleration, assuming it to be constant over that time period and giving maximum weight to the most recent estimate.

$$
\begin{aligned}
\hat{\mathbf{a}}_{p}(k) & =\frac{1}{T} \sum_{i=1}^{m} c_{i}\left(\mathbf{v}_{p}(k-i+1)-\mathbf{v}_{p}(k-i)\right) \\
\sum_{i=1}^{m} c_{i} & =1
\end{aligned}
$$

While the number of past points used, and the filter coefficients can be chosen to optimize some objective function, we simply chose $m=5$ and $c_{1}=\frac{17}{32}, \quad c_{2}=\frac{1}{4}, \quad c_{3}=\frac{1}{8}, \quad c_{4}=\frac{1}{16}, \quad c_{5}=\frac{1}{32}$. Using the estimate of the acceleration, we perform prediction of the current state of the quarry (position and velocity) using the quarry double integrator model:

$$
\begin{aligned}
\left(\begin{array}{c}
\hat{\mathbf{x}}_{p} \\
\hat{\mathbf{v}}_{p}
\end{array}\right)(k) & =\mathbf{A}^{m}\left(\begin{array}{c}
\hat{\mathbf{x}}_{p} \\
\hat{\mathbf{v}}_{p}
\end{array}\right)(k-m)+\sum_{i=1}^{m-1} \mathbf{A}^{i} \mathbf{b} \hat{a}_{p} \\
\mathbf{A} & =\left(\begin{array}{cc}
\mathbf{I}_{2} & T \mathbf{I}_{2} \\
\mathbf{0} & \mathbf{I}_{2}
\end{array}\right), \quad \mathbf{b}=\left(\begin{array}{c}
\mathbf{0} \\
T \mathbf{I}_{2}
\end{array}\right)
\end{aligned}
$$

Finally, we update the vertical coordinate of the aerial vehicle with the following gradient descent type law that minimizes the cost function

$$
J=2 \frac{\left\|\delta \mathbf{x}_{p l}\right\|^{2}}{z_{c}^{2} \tan ^{2} \frac{\alpha}{2}},
$$

with respect to $z_{c}$, giving

$$
\dot{z}_{c}=4 \frac{\left\|\delta \mathbf{x}_{p l}\right\|^{2}}{z_{c}^{3} \tan ^{2} \frac{\alpha}{2}}
$$

The above cost function is motivated by the idea of maintaining the position of the aerial vehicle and therefore its tracking camera, within a square inscribed inside the field-of-view circle on the ground. Besides, it ensures that the vehicle does not climb so high that it can no longer detect the quarry. Most chase missions are of short duration, so the constant increase of altitude does not take the UAV too high.

\section{RESUlts}

We performed simulations with the above control law on a representation of the Ft. Benning MOUT site with both a hover capable chaser and a fixed wing chaser (can't go slower than a minimum velocity). The vehicle capabilities were as follows: maximum speeds of $25 \mathrm{~m} / \mathrm{s}$ (hover-capable) and $40 \mathrm{~m} / \mathrm{s}$ (fixed wing), maximum acceleration of $10 \mathrm{~m} / \mathrm{s}^{2}, \tau_{x}=0.25 \mathrm{~s}, \tau_{v}=0.5 \mathrm{~s}$, the minimum speed for the fixed wing vehicle was $25 \mathrm{~m} / \mathrm{s}$, and the vehicle had flew between 25 and $50 \mathrm{~m}$. The field of view of the camera was taken as $\alpha=120$. The two figures below show the chase results for the hover capable and fixed-wing vehicles. The quarry in each case is denoted by solid circles at times of 1,2,3,7,14,21,28 seconds (moving from left to right on the map in the figures). The chaser is represented by an unfilled circle at those same times. The path of the quarry is represented by a thick black line and the path of the chaser is represented by a thin line. The target vehicle is always either accelerating or decelerating at $3 \mathrm{~m} / \mathrm{s} 2$. It has a maximum velocity of $25 \mathrm{~m} / \mathrm{s}$ (almost 60mph) and it negotiates turns with a velocity of $7.5 \mathrm{~m} / \mathrm{s}$. The measurement noise variance for both position and velocity is 5 units (very large compared to actual values).

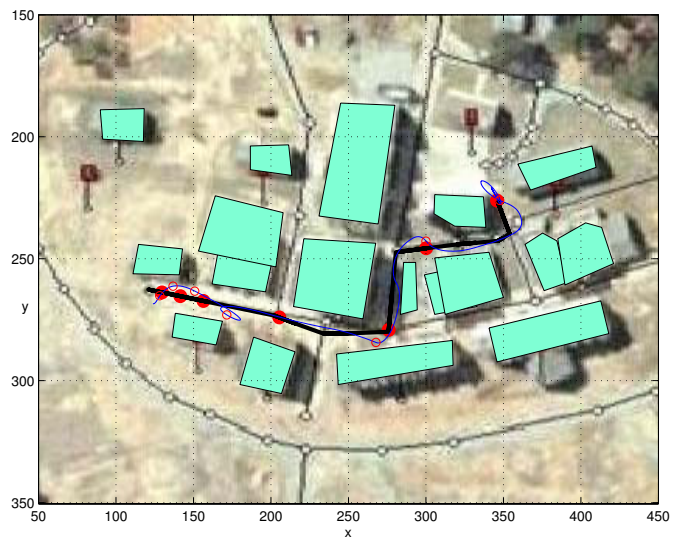

Fig. 2. Chase by a hover capable UAV

Figure 2 shows the chase done by the hover capable vehicle. Figure 3 shows the altitude variation of the chaser during the chase. In this case, the chaser is able to maintain its quarry in view while it follows it. The altitude remains in a small range-which is desirable as we don't want vehicle actuation authority to be wasted in altering altitude except when necessary. Figure 4 shows the corresponding chase for a fixed-wing chase vehicle and Figure 5 the variation in its altitude. Since this cannot fly slower than $25 \mathrm{~m} / \mathrm{s}$, it has to make figure of eights and circle around the quarry as the quarry slows 


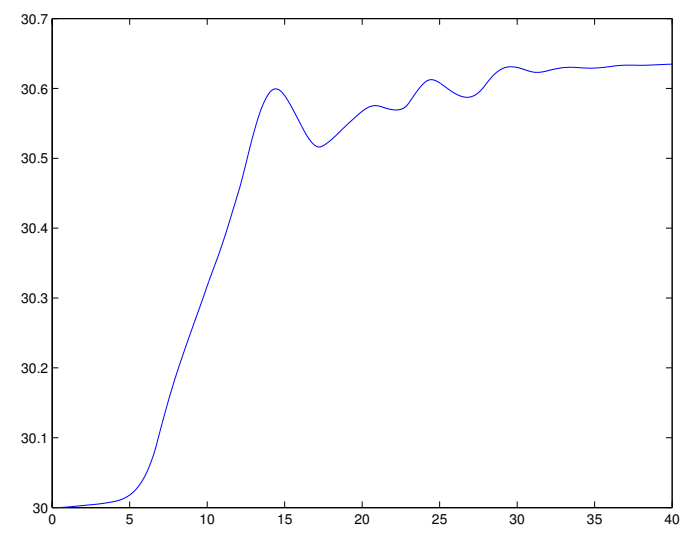

Fig. 3. Vertical motion of the hover capable UAV

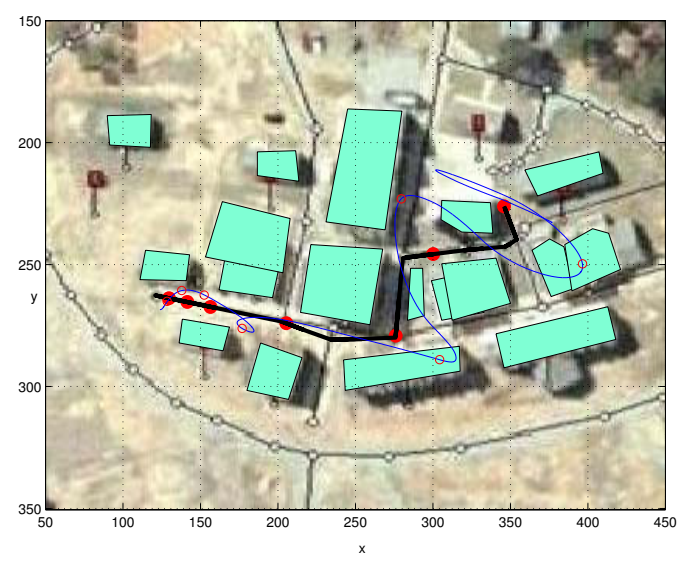

Fig. 4. Chase by a fixed wing UAV

down at the turns. It also has to climb a bit more to prevent the quarry from giving it a slip.

\section{CONCLUDING REMARKS}

The chaser as is locally exponentially stable by design-as the $\mathrm{x}$ and $\mathrm{y}$ position error equations are exponentially stable, and the gradient descent is also stable (the altitude cannot increase indefinitely). We may be able to determine a stability region of the tracking in the presence of vehicle constraints such as predator or prey vehicle acceleration and speed limits using Sum of Squares programming [3]. Performance in the presence of noise and occlusions (no measurement for a few time steps) is also amenable to analysis in the Sum of Squares framework.

\section{REFERENCES}

[1] R. Isaacs, Differential Games: A Mathematical Theory with Applications to Warfare and Pursuit, Control and Optimization, Dover Publications, Mineola, NY, 1999.

[2] J. Lee, R. Huang, A. Vaughn, X. Xiao, J.K. Hedrick, M. Zennaro and R. Sengupta, "Strategies of Path-Planning for a UAV to Track a Ground vehicle," In Autonomous Intelligent Networked Ssystems Workshop, Menlo Park, CA June 2003. http://path.berkeley.edu/ains/final/002

[3] S. Prajna and A. Papachristodoulou and P. Seiler and P. A. Parrilo, SOSTOOLS: Sum of squares optimization toolbox for MATLAB, Available from http://www.cds.caltech.edu/sostools, and http://www.mit.edu/ parillo/sostools, 2004.

[4] U. Zengin and A. Dogan, "Target tracking by UAVs under Communication Constraints in an Adversarial Environment," AIAA GNC Conference, August 2005, San Francisco, CA

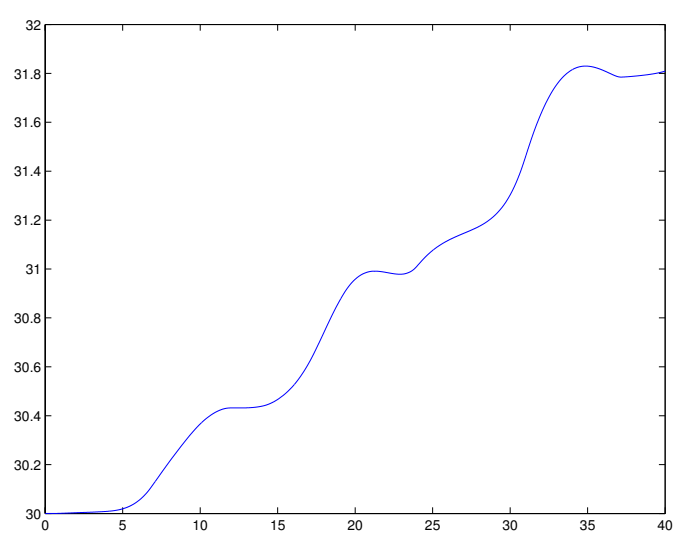

Fig. 5. Vertical motion of the fixed wing UAV 